\title{
The Diagnosis \& Management of Non-Carious Cervical Lesions by Dental Practitioners from the South Yorkshire and Bassetlaw Regions of England
}

\section{El-Dhuwaib B*}

Clinical Teacher in Restorative Dentistry, University of Sheffield, England

*Corresponding author: Bilal El-Dhuwaib, BDS, MJDF DipRestDent FFGDP RCS Eng, MSc, PgCert HC Ed., General Dental Practitioner in South Yorkshire, Regional Clinical Director for Dental Partners, and a Clinical Teacher in Restorative Dentistry, School of Clinical Dentistry, University of Sheffield, United Kingdom, England, Tel: 07498869160; Email: bilal.el-dhuwaib@ sheffield.ac.uk

\section{Research Article \\ Volume 7 Issue 1}

Received Date: January 03, 2022

Published Date: January 18, 2022

DOI: $10.23880 /$ oajds-16000321

\section{Abstract}

A survey-based study, utilising qualitative and quantitative questions was used to investigate the diagnosis and the management of non-carious cervical lesions (NCCLs), including an overview to the working and gender demographics of primary dental care practitioners in South Yorkshire. To establish the contributing factors associated with NCCLs; to identify the most common approach used by dentists on how to manage NCCLs, and to identify what restorative materials dentists used and why.

Objectives: To investigate, by the use of a questionnaire, the diagnosis \& management of non-carious cervical lesions by dental practitioners.

Materials and Methods: A sample size calculation yielded a survey size of 149, following initial returns (non-responders) the questionnaire was posted to 300 randomly chosen participants. The questionnaire contained an initial demographic section to ascertain; gender, time since graduation and working times (8 questions), and the second section focused 6 questions upon; NCCL diagnosis, management and treatment options. Likert scale (5 point responses: greatly agree to greatly disagree), freetext boxes, closed-ended and multiple-choice questions were used to collect data from respondents. The response rate was (53.3\%).

Results: Abrasion, particularly tooth brushing, was believed to be the main cause of NCCLs (87\%). An initial monitor/ preventative (fluoride application) approach was taken first, with the rationale to restore being based upon patient reported symptoms, first, and then followed by any aesthetic concerns/ preservation of tooth tissue. When NCCLs were being treated there was a wide range of numbers: modal value of 2, and median value of 5 NCCLs being restored per week. Composite was the aesthetic material of choice to restore NCCLs (57\%), followed by (30\%) for glass polyalkenoate (GI cements) restorations. Conclusion: Our demographic data indicated that the survey cohort could be considered as a representative sample of primary care dental practitioners in terms; of gender, time since qualification (as a proxy of age), and working practice (NHS vs. Private), and sessions worked per week. There was a strong agreement between dentists that tooth brushing abrasion is the main causes of NCCLs., and while the majority of NCCLs are managed conservatively, composite is the most frequent restorative material used by dentists to restore NCCLs followed by GIC. Dentists are more likely to restore NCCLs to improve sensitivity or to preserve remaining tooth structure, than to improve patients' aesthetics. The size of the cavity, the anatomical position of the tooth, the nature of the cause, the aesthetic consideration and the materials' technical properties had an effect on deciding the choice of restorative material.

Keywords: Non-Carious Cervical Lesions 
Abbreviations: NCCL: Non-Carious Cervical Lesions;

\section{Introduction}

Successive UK Adult Dental Health Surveys, over a 32-year time period [1-3], shows a decreasing trend of edentulousness, with a subsequent increase in tooth retention (Chart 1). However, examination of the relative distributions of tooth retention vs. age also shows a decreasing trend of tooth retention with age (Chart 2). Tooth surface wear is seen to increase with age (Chart 3) although this reported wear is on all surfaces, rather than identified by specific sites, so includes the conditions; abrasion, attrition, erosion and abfraction. This study focuses upon non-carious cervical lesions (NCCL), with the reported prevalence of NCCLs increasing with age [4-10] and are seemingly a relatively common clinical finding.
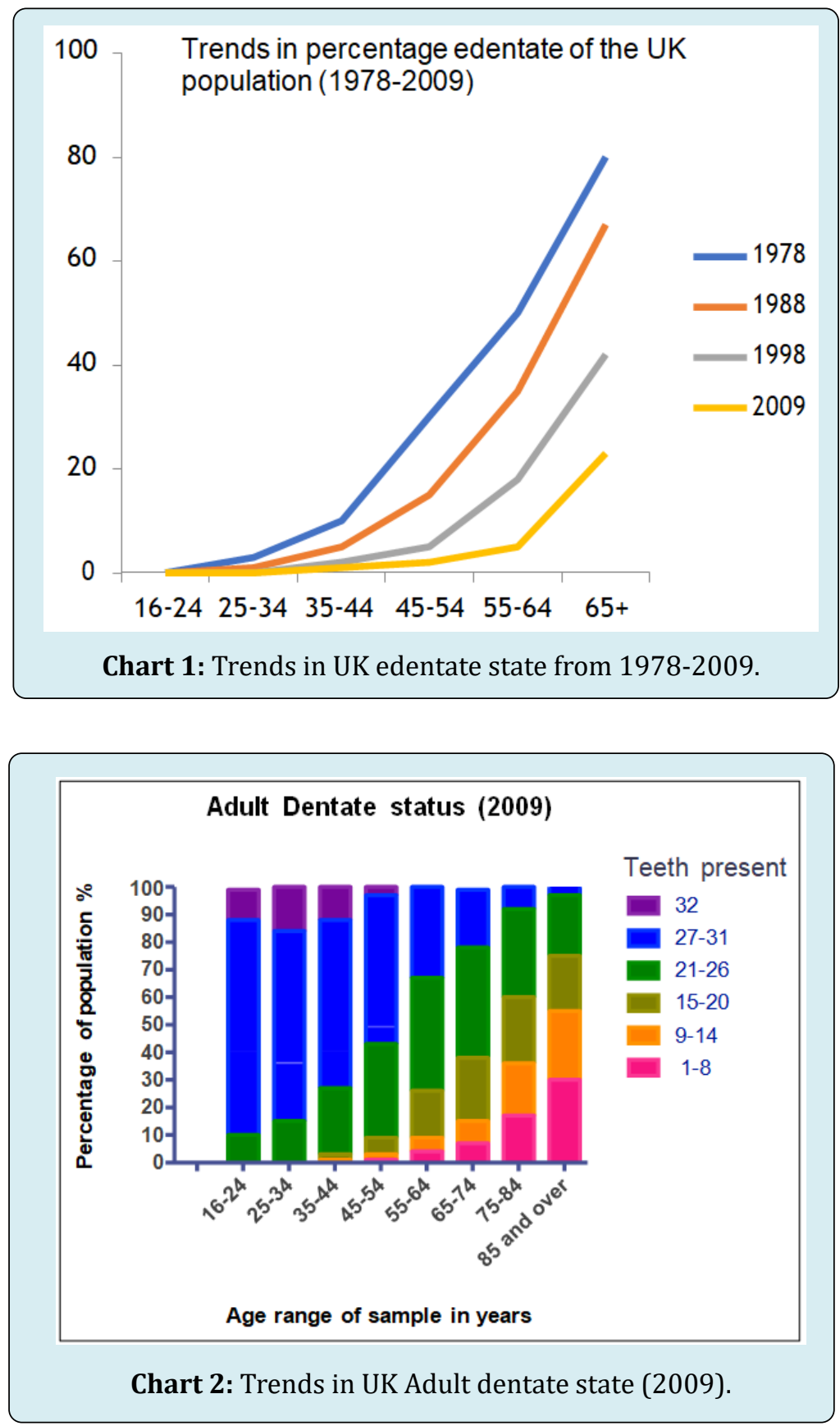


\section{Open Access Journal of Dental Sciences}

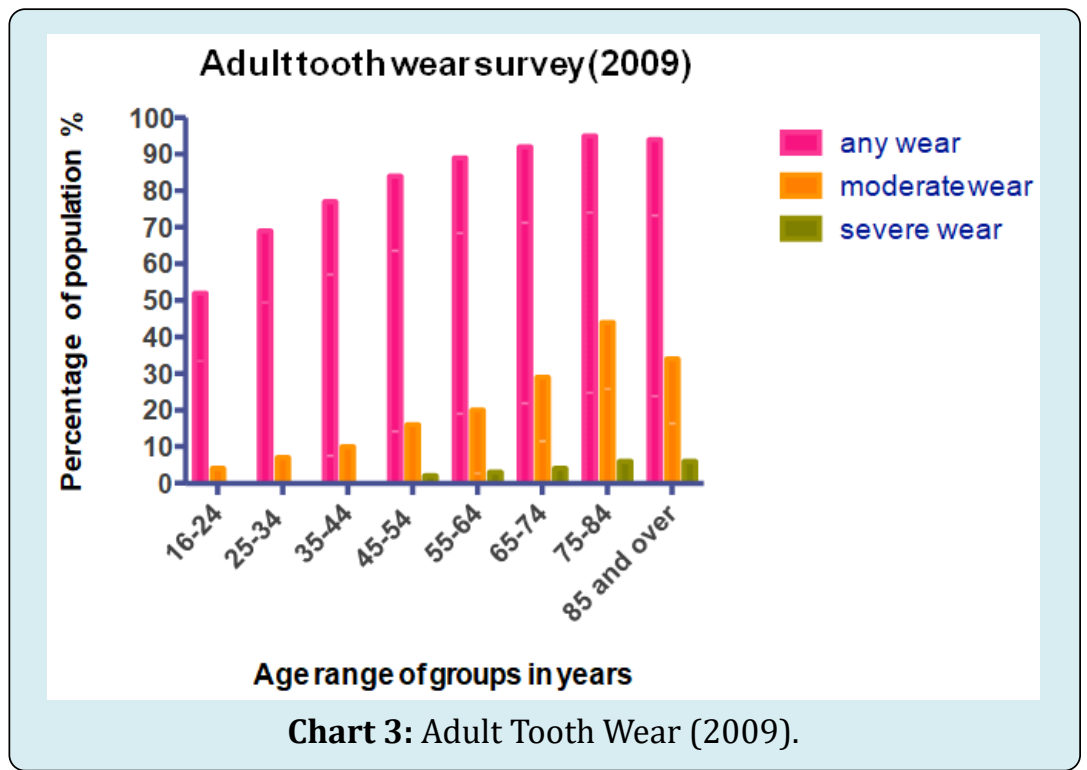

In 2008 a Literature Review of NCCCLs [11] indicated a prevalence range from $2-90 \%$, and this variation is thought to be due to; "Difference in definition of NCCL, age, and cultural and behavioural differences within the research sample." NCCLs are commonly found on upper premolars [11-14] with the following factors thought to play a part in their formation (Table 1).

\begin{tabular}{|c|}
\hline Age \\
\hline Gender \\
\hline Anatomical tooth location \\
\hline Right or left sided dominance \\
\hline Hardness/ rigidity of tooth brush bristles \\
\hline Dentifrice abrasiveness \\
\hline Frequency/ intensity of toothbrushing \\
\hline Occlusal loading/ bruxism \\
\hline
\end{tabular}

Table 1: Factors implicated in developing NCCLs.

Researchers have concluded that NCCLs might be restored to: reduce sensitivity, maintain structural integrity, protect pulpal vitality, reduce plaque retention, improve retention of partial dentures, and restore aesthetic harmony [12,15-17]. Non-carious cervical lesions are believed to be a multifactorial process, initiated when one or more factors interact to develop these lesions $[16,18,19]$. Interestingly, the initiating factors for a NCCL may be different to the factors causing further lesion development $[20,21]$.

\section{Methodology}

For this MSc study, a postal questionnaire was considered the most efficient way of gathering quantitative, and qualitative data from dental practitioners on their diagnosis and management of NCCLs. A master list of the names and addresses of primary dental care practitioners in the South Yorkshire and Bassetlaw area was sourced from: on-line General Dental Council Register (2013), local Primary Care Trusts, NHS Choices and Dental Practice websites, with Orthodontic only practices excluded from the survey. The master list comprised 604 individuals, and a sample size calculation (www.raosoft.com) determined a survey sample of 149 dental practitioners (5\% margin of error \& 95\% confidence level \& $50 \%$ distribution rate). The master list was arranged alphabetically, sequentially numbered, and then a random number generator (www.random.org) created a list of 300 random numbers, from which practitioners could then be chosen from the master list.

The questionnaire, 3 pages of A4 in total, was composed of two main sections: Part 1 individual demographic data e.g. gender, country of graduation with a dental degree, time in practice (5 year cohorts), NHS vs. Private practice, workload related to NCCL \& postgraduate qualifications (8 questions). Part 2 looked at the diagnosis, management and materials used in the restoration of an NCCL (6 questions). Part 1 collected quantitative data; with Part 2 collecting qualitative data (opinions or views) utilising structured questions, and answered using a 5-point Likert scales; strongly agree, agree, neutral, disagree \& strongly disagree, with available free text comment space for each question.

These questions were to ascertain any contributing factors that might be associated with non-carious cervical lesions such as; gender of the patient, positional relationship of NCCL to the dominant hand of the patient, toothbrushing/ toothpastes, and/or occlusal forces. Questions were also asked of participants to identify their reasons behind 
restoring these lesions, with their approach on how to manage them, including, their considered 'best' restorative material. The questionnaire was constructed, trialed in Dinnington Dental Practice, and then refined over 8 versions, before The University of Leeds School of Dentistry ethical approval process, and its subsequent approval.

A research pack was then posted out to the chosen random sample individuals, with a covering letter introducing the researcher and to explain the research. This covered their involvement with it, or not, measures taken to protect the confidentially of their data, a copy of the questionnaire, and a self- addressed paid envelop to return the completed questionnaire. The questionnaire asked for no identifiable personal information, and was only numerically identified to the sample list on a plain cover sheet on the questionnaire, allowing cross checking of questionnaire returns, for resending a reminder pack (after 3 weeks), or taking new names from the master list as required to reach the sample size returns. A member of practice staff was allocated the task of crosschecking and tallying returns and reminders, and once returned the identifier sheet was destroyed to preserve individual anonymity.

The master list, randomisation list and paper questionnaire returns were all stored in a locked filing cabinet until the data entries were made, checked and verified, before secure shredding of the questionnaires. Data was entered into a Microsoft (C) Excel spreadsheet, and with participant comments/ opinions or views entered into a Microsoft (C) Word document, and with all digital data stored in an encrypted file, with access restricted to the Authors. Statistical analysis used Prism 6 (C) 1995-2017 GraphPad Software, Inc.) statistical package for Mac (C) Apple Computers).

\section{Results}

\section{Return-rate and GenderAnalysis OfRespondents}

The Master list contained 604 possible participants, with 160 / 300 returned questionnaires; a return rate of $53 \%$, and a sampling rate of approximately 1 in 4 achieved. 95\% of questionnaires were returned completed, $2 \%$ partially completed, and 3\% uncompleted.

\section{Gender Analysis Of Respondents}

Gender data (Chart 4) was collected in 5-year cohorts, from time since graduation, and then grouped into percentage ratios. Four cohorts, Years 1-5, 11-15, 16-20 \& 21-25 (1989-2013) have male ratios within a range of 54$60 \%$, and female ratios within $40-46 \%$. Three cohorts, Years 26-30, 31-35, 36-40 (1974-1988) are very male dominated, with male ratios within a range of $82-88 \%$, and female ratios within $12-18 \%$. In cohort 6-10 there is seemingly a gender reversal, with a male to female ratio of 42: 58\%. A more equal gender distribution in the cohorts Years 1-25 (1989-2013) is indicative of increased levels of female undergraduate recruitment.

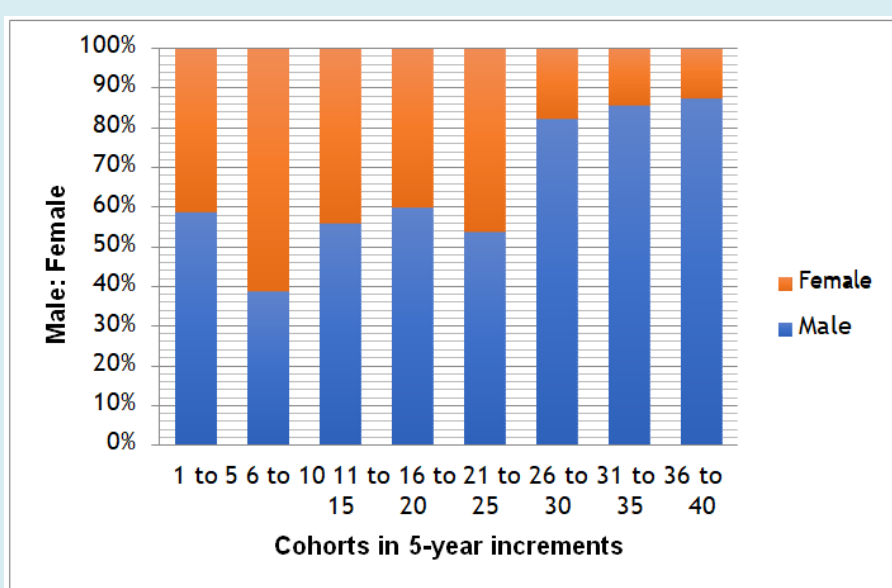

Chart 4: Gender analysis of respondents by 5-Year cohorts since graduation.

NHS Dental Statistics for England [22] data (Chart 5) shows that overall the male to female dentist ratios have dramatically changed from the period 2006-07 (61:39\% M to $\mathrm{F}$ ratio) to $2016-17$ (51:49\% $\mathrm{M}$ to $\mathrm{F}$ ratio). Chart 5 shows this increasing trend of female to male dentist ratios in all cohorts; <35 years of age, 35-44, 45-55 \& above 55 years of age since $2006-07$. 


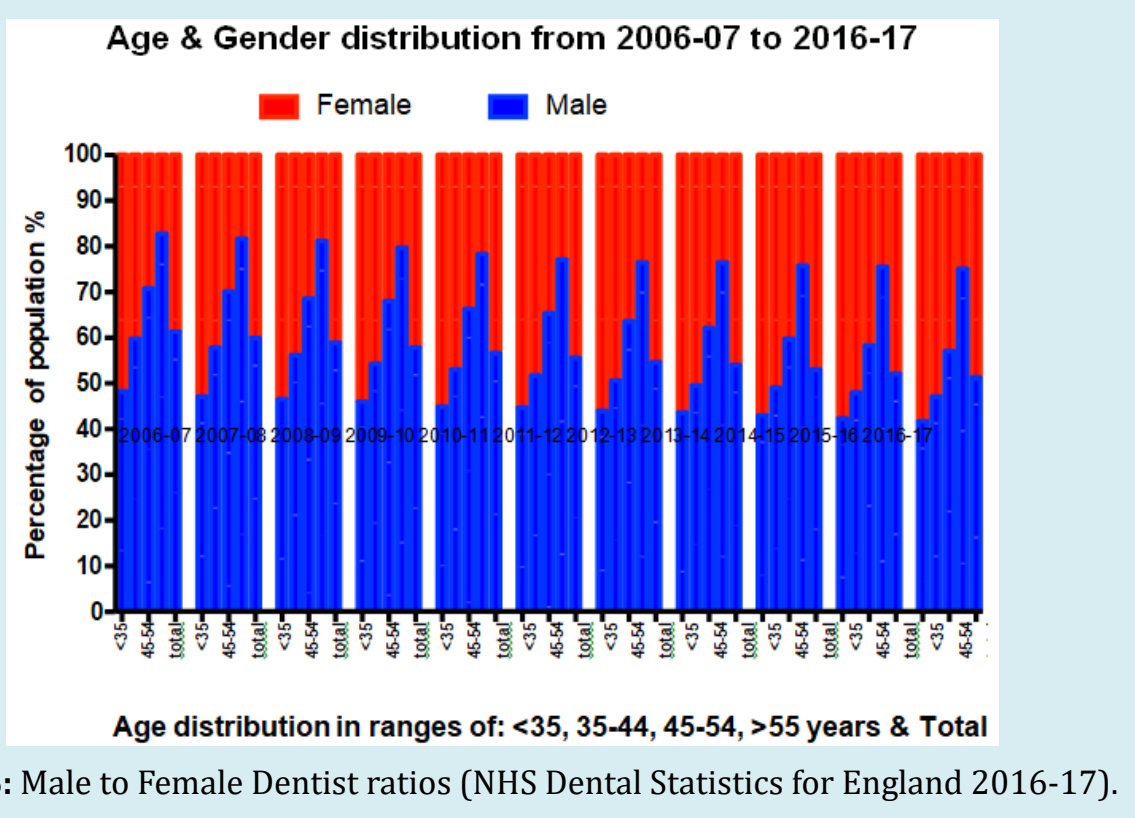

\section{Practice Profiles of Respondents: Clinical Practice- NHS To Private Ratios}

NHS practice is predominant among our respondents (Chart 6), with a much lower level of private work being carried out overall. On average $84 \%$ of services was undertaken by NHS contract while $16 \%$ is under a private services contract. The most frequent mode of contract was $95 \%$ NHS services and $5 \%$ private service.

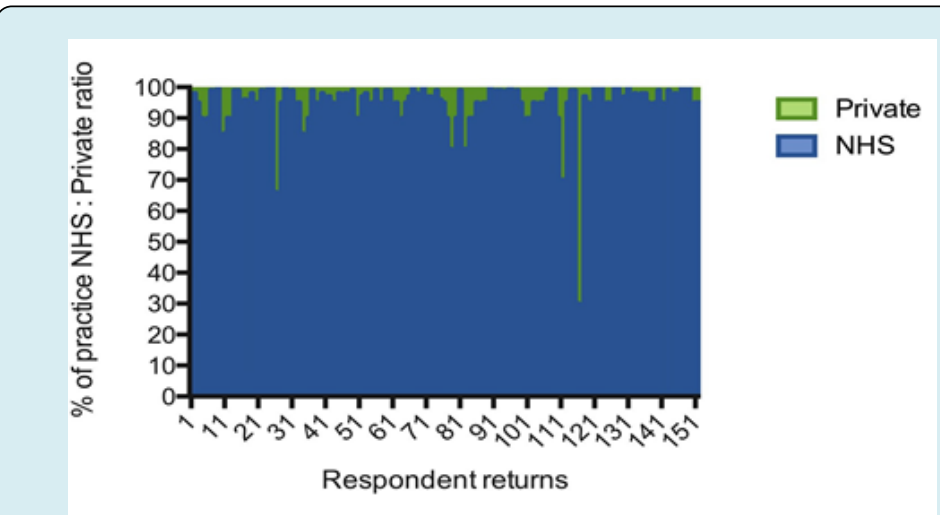

Chart 6: The clinical practice of respondents- NHS to Private ratio.

\section{Practice profiles of respondents: Working time (F/T: P/T)}

Respondents were asked about the number of clinical session worked routinely per week, with one session being either a morning or afternoon period, and this was reported at working between 1 to 11 sessions a week (Chart 7). The median reported value was 8 sessions a week, or 4 full days, for both male and female respondents. The $75 \%$ percentile value was 10 sessions for males, and 9 sessions for females, with overall $69 \%$ of respondents working at least 8 sessions or more. 


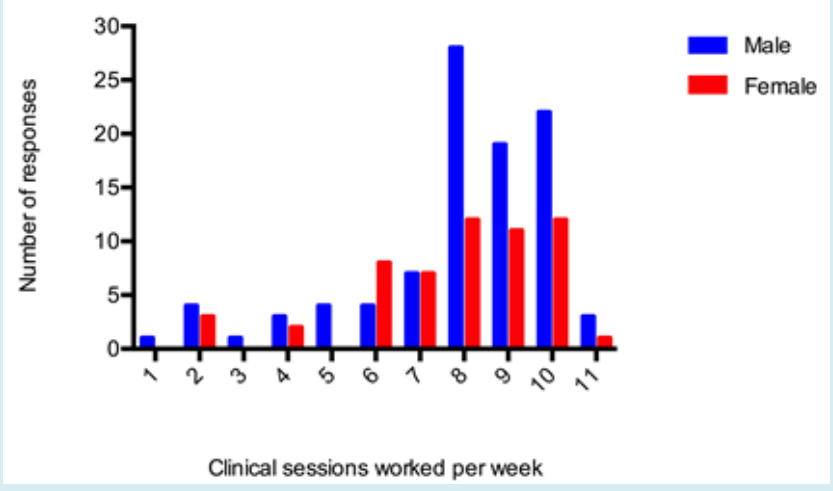

Chart 7: Histogram of working profiles of male: female and $\mathrm{F} \backslash \mathrm{T}$ : $\mathrm{P} \backslash \mathrm{T}$ sessions.

There are minimal differences, statistically insignificant (Table 2), from this survey into clinical working sessions due to gender.

\begin{tabular}{|c|c|c|c|c|}
\hline & Median & Mean & SEM & Unpaired t-test \\
\hline Male & 8 & 7.96 & 0.23 & $\mathrm{P}=0.64$ \\
\hline Female & 8 & 7.8 & 0.28 & \\
\hline
\end{tabular}

Table 2: Statistical analysis of gender and clinical sessions worked per week.

\section{Factors implicated in the causation of NCCLs}

This question used a 5-point Likert scale (Range: strongly disagree (1) to greatly agree (5)) and respondents were asked about what they believed were the most commonly related NCCL factors such as; gender (Male vs. Female), occlusal factors (i.e. abfraction) tooth brushing technique (i.e. abrasion), dominant hand/ contra lateral side of mouth. Data was analysed using one-way ANOVA, and then represented graphically by box \& whisker plots (Chart 8).

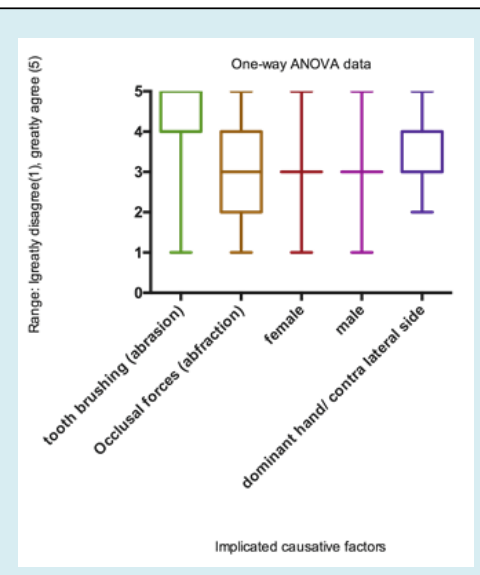

Chart 8: Implicated causative factors for NCCL development by respondents.
Tooth brushing (abrasion) is seen as the most likely causative factor for NCCLs (interquartile range 4- 5), followed by dominant hand/ contra lateral side for tooth brushing (interquartile range 3-4). The results for gender (M \& F) show a relatively neutral set of views (interquartile range 3) i.e. a neutral outlook, whereas the question of occlusal forces in generating NCCLs (abfraction) is more diverse (interquartile range 2-4) from agree to disagree. This is not unsurprising, as the question over the existence of occlusal force generating abfraction lesions is in its self a somewhat contentious issue within clinical circles.

\section{Management of NCCLs by the respondents}

The respondents were asked to rank seven possible ways of managing a non-carious cervical lesion scenario (Table 3), in order from 1-7 (where 1 is the most likely approach and 7 is the least likely approach of management) to a buccal sited NCCL, on a maxillary first premolar tooth, that is at least 1.5 to $2 \mathrm{~mm}$ in depth.

\begin{tabular}{|c|}
\hline Monitor \\
\hline High fluoride application \\
\hline Restore with a composite resin restoration \\
\hline Restore with a resin modified glass ionomer or a glass \\
ionomer cement \\
\hline Restore with a compomer restoration \\
\hline Restore with amalgam \\
\hline Advanced restorative option: veneer or crown \\
\hline
\end{tabular}

Table 3: Different ways of management of NCCLs.

The data was analysed using Prism 6 statistical software using one-way ANOVA test, and graphically represented using bar and whisker plots (Chart 9) to reveal the trends in management decision- making among the respondents. 


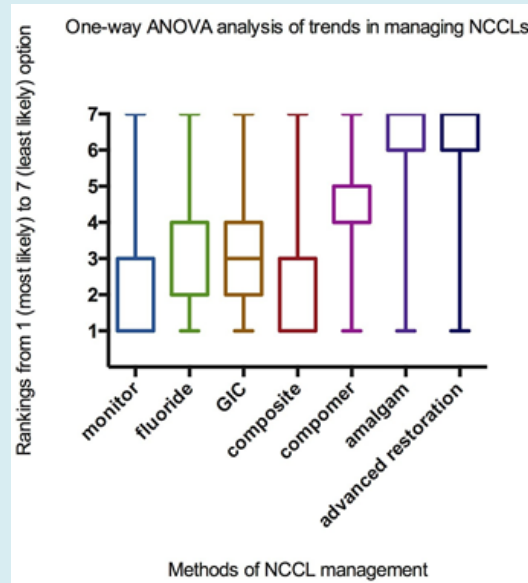

Chart 9: The management of a scenario NCCL by respondents.

The trends in management of a conceptual NCCL lesion show that the respondents were most likely (1-3 interquartile rankings) to either monitor, or to place a composite resin restoration. At $1.5-2 \mathrm{~mm}$ in depth this NCCL would be ideal for an adhesive, non-preparation technique, with composite resin.

Second most popular options (2-4 interquartile rankings) were again both non-preparation approaches, utilising either a topical fluoride application, or an adhesive glass polyalkenoate cement (glass ionomer cement) restoration.

Least likely approaches (6-7 interquartile rankings) involved the placement of amalgam, which would require tooth preparation and be highly unaesthetic, or the preparation for an advanced restoration that would require extensive tooth preparation, and therefore be unwarranted in this concept scenario. Interestingly, compomer resins, (4-5 interquartile ranking) would seem to be the least ideal choice from the list of aesthetic restorative materials, and may represent a material that offers benefits of composite and admixed glass polyalkenoate cement not performing as well under real life clinical conditions, as in laboratory simulation.

Free comment text boxes were provided to allow respondents to further expand upon their replies, if they so wished, and five broad themes were identified for the choice of restorative material:

a) The size of the cavity; with composite restorations for cavities $>1 \mathrm{~mm}$ (medium to large) and the use of GIC restorations for those $<1 \mathrm{~mm}$ in depth.

b) The specific tooth affected; with composite restorations for incisors and premolars, while GIC for molars.

c) The nature of the cause; with composite for abrasion and GIC for abfraction cavities. d) The aesthetic properties of the restorative material, with amalgam considered by some GDPs as the most durable material, but its poor aesthetic quality limits its use.

e) The restorative technique required to use the different restorative materials can be a significant factor in selecting what restorative material can be used. Preparing a retentive cavity for an amalgam restoration requires tooth removal and this is not always feasible or advisable. Restoring a cavity with composite restoration necessitates a good moisture control, and in situations with poor moisture control, a GIC may be the material of choice.

\section{Reasons for restoring a NCCL}

This question used a 5-point Likert scale (Range: least likely (1) to most likely (5)) to analyse why respondents may restore NCCLs lesions, based upon 3 specific patient outcomes questions: has aesthetic concerns, reports sensitivity, or general patient request, and 1 clinician driven question: to further preserve tooth substance. This was analysed using repeated measures ANOVA, and is summarised in Chart 10 below.

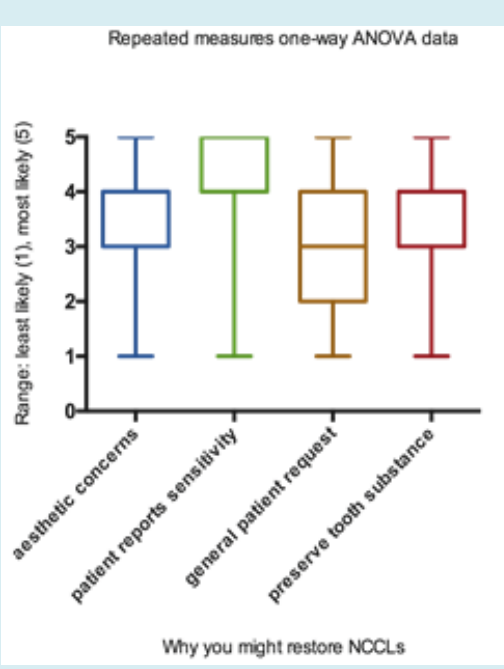

Chart 10: Why respondents might restore a NCCL.

The primary reason for restoring a NCCL lesion show that respondents were most likely/ likely (4-5 interquartile rankings) to restore when a patient reported sensitivity, followed secondly by an equal spread of responses neutral/ likely (3-4 interquartile rankings) for aesthetic concerns or to further preserve tooth substance. General patient request had a split response across unlikely / neutral/ likely to restore a NCCL (2-4 interquartile rankings).

The participants were also asked to estimate the number of restorations they provided to restore NCCLs in an average week (Chart 11), with a mode value 2 , median value 5 , and a 
mean value of 6 restorations per week.

Histogram of Frequency distribution data

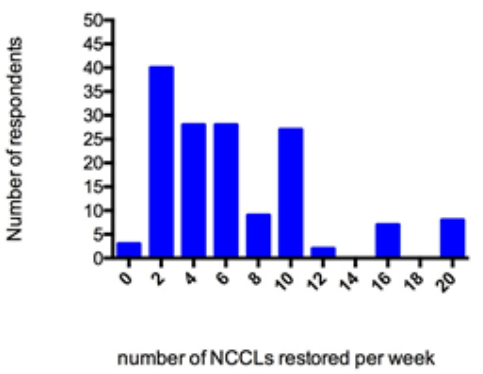

Chart 11: Estimated number of NCCLs restored per week

\section{Conclusion}

\section{Survey Response, Respondent Gender \& Working Time Practice}

The response rate for this study was $53.3 \%$, positioned between those of Bader, et al. [18] at 47\%, and Lyttle et al. [12] at $63 \%$. We had a sampling rate of approximately 1 in 4 of the potential dentist population achieved. Survey-based questionnaires can provide valuable data for researchers, covering quite large geographical areas, and with a larger number of contacts possible than when face-to-face interviews are used. However, the use of a survey has a number of advantages [22], but also disadvantages inherent in its use (Table 4).

\begin{tabular}{|c|c|}
\hline Advantages & Disadvantages \\
\hline $\begin{array}{c}\text { Scalability, from pilot trial, through small scale surveys to } \\
\text { large geographical area surveys }\end{array}$ & Lack of personalisation, can lead to respondents not replying \\
\hline $\begin{array}{c}\text { Large numbers of respondents can be contacted- } \\
\text { representation of your population \& a cost effective method }\end{array}$ & $\begin{array}{c}\text { Potential for low response rates if participants are not engaged } \\
\text { in the topic, and then statistical validity of the result is low }\end{array}$ \\
\hline Relatively simple to construct & $\begin{array}{c}\text { May lack complexity, or be too complex or overly long, and it } \\
\text { lacks the nuances that face- to-face interviews offer, and cannot } \\
\text { be modified in light of answers / respondent questions in } \\
\text { returns }\end{array}$ \\
\hline $\begin{array}{c}\text { Can use published Likert scales \& questions from other } \\
\text { surveys for reproducibility/ validity. Data can be easily } \\
\text { extracted, and allowing quantitative statistical analysis. }\end{array}$ & $\begin{array}{c}\text { Unless Analysis is planned into the question design, then the } \\
\text { data analysis may be meaningless }\end{array}$ \\
\hline $\begin{array}{c}\text { Random allocation, numbering of questionnaires, and third } \\
\text { party collation minimises any researcher bias }\end{array}$ & $\begin{array}{c}\text { Response bias: topic appeals to the participant (reply) or not } \\
\text { (no reply). }\end{array}$ \\
\hline Quick time frame for response and data acquisition & \\
\hline
\end{tabular}

Table 4: Advantages and disadvantages of survey [22].

The gender profile, within the limitations of this survey, would seem to follow a similar trend seen in the national gender profile for dentists from NHS England, and the age/ gender profiles would also fit within historical UK trends. This is indicative of the increased recruitment of female undergraduates seen over the last 30 years into UK Dental Schools.

Our survey respondents were predominantly NHS practitioners, on average undertaking 84\% NHS service and $16 \%$ under a private services contract, with the modal value of $95 \%$ NHS services and 5\% private service. Respondents were generally working 8 sessions (median value), and there was no statistically significant difference between the genders when this was was factored in. It would seem from these results that our sample was representative of the dentist population of the geographical area of this survey.

\section{Factors Implicated in the Causation of NCCLs}

The research evidence is quite equivocal over the different factors implicated in NCCLs, as they are viewed as being multifactorial in nature $[4,5,11,16,23-25]$ with a fairly long development time-line. Our results see toothbrushrelated abrasion being the most highly rated factor for a NCCLs, and with them being mostly seen on the dominanthand side of the mouth, with gender, and occlusal forces (abfraction) not seen as predominant factors.

\section{When, Why and How To Restore NCCLs}

Our respondents indicated that they would restore between 2 (modal value) to 10, with a median value of 5 NCCLs per week, so that this shows a relatively low workload associated with NNCLs, although a minority were restoring much higher numbers (10-20 NCCLs). When asked about 
reasons to restore NCCLs our respondents took a monitor/ preventative (fluoride application) approach, with a rationale to restore based upon patient reported symptoms, first, and followed by aesthetic concerns/ preservation of tooth tissue.

The restorative techniques again are based upon the use of minimal intervention dentistry and adhesively bonded composites, followed in choice by glass ionomer cements. This is interesting, in that NCCL cavitation can have rather sclerosed dentinal tubules, and burnished dentine, which is less than ideal for physico-chemical interlocking in dentine bonding, whereas glass ionomer bonding to dentine via long chain carboxyl groups would theoretically be a better bond. However, the physical characteristics/ surface polish/ colour matching of a composite resin is superior to that achievable by glass ionomer cements, in what is generally an aesthetic region of the mouth. This trend of favouring composite over GIC has been found to be consistent with other studies $[12,17]$, and Chart 12 summarize the results.

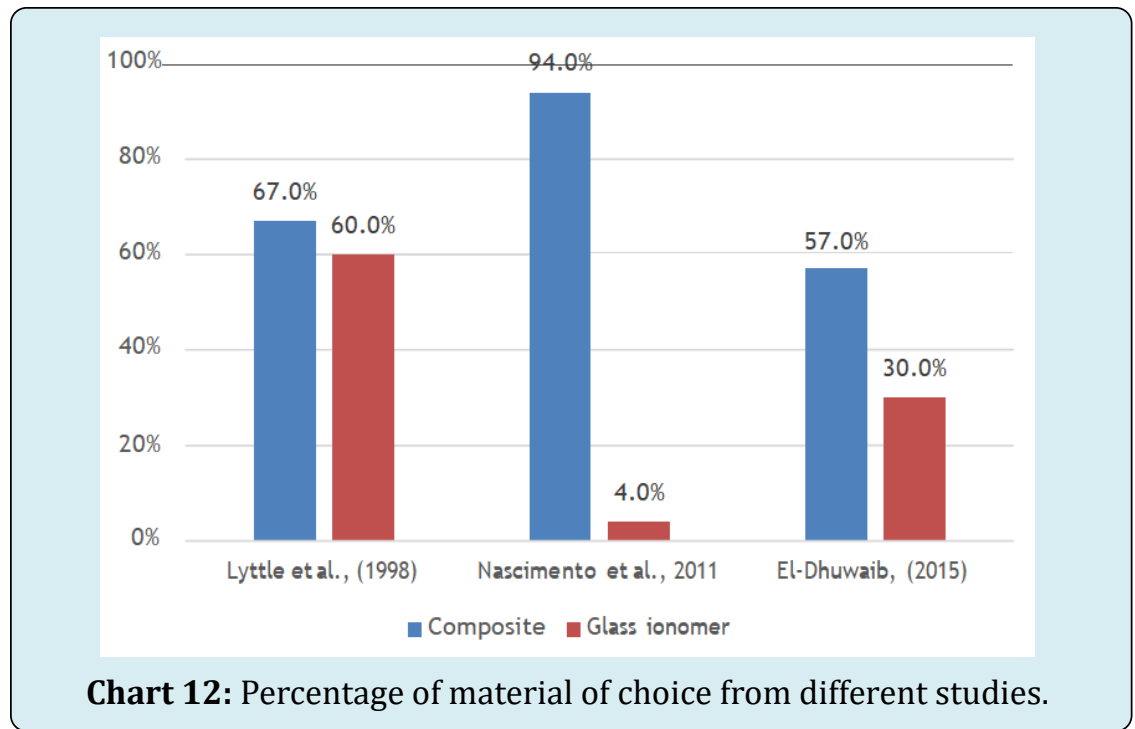

\section{Summary}

We, as clinicians, tend to notice the 'end result' of NCCLs, as clinically observable cavitation or loss of the tooth surface. This makes the dissection out of those different factors, with their potential interaction/ time ordering/ and intervals difficult to discern. Sadly this makes it almost impossible to carry out a relevant patient-centred clinical research project into the actual process of NCCLs development and progression.

However, this research shows that the practitioners involved were taking a pragmatic approach, based upon a preventative regimen, and where applicable using minimal interventionist techniques to the management and restoration of NCCLs, which is most admirable and to be highly commended.

\section{References}

1. Office for National Statistics (2011) Adult Dental Health Survey 2009-Summary report and thematic series. NHS, Adult Dental Health Survey.

2. Office for National Statistics (1998) Social Survey
Division. Adult Dental Health Survey. $2^{\text {nd }}$ (Edn.), UK Data Service.

3. Office of Population Censuses and Surveys. Social Survey Division. Adult Dental Health.

4. Telles D, Pegoraro L, Pereira J (2000) Prevalence of Noncarious Cervical Lesions and Their Relation to Occlusal Aspects: A Clinical Study. J Esthet Dent 12(1): 10-15.

5. Bernhardt O, Gesch D, Schwahn C, Mack F, Meyer G, et al. (2006) Epidemiological evaluation of the multifactorial aetiology of abfractions. J Oral Rehabil 33(1): 17-25.

6. Takehara J, Takano T, Akhter R, Morita M (2008) Correlations of noncarious cervical lesions and occlusal factors determined by using pressure-detecting sheet. J Dent 36(10): 774-779.

7. Smith WA, Marchan S, Rafeek RN (2008) The prevalence and severity of non-carious cervical lesions in a group of patients attending a university hospital in Trinidad. J Oral Rehabil 35(2): 128-134.

8. Wood ID, Kassir ASA, Brunton PA (2009) Effect of Lateral 
Excursive Movements on the Progression of Abfraction Lesions. Oper Dent 34(3): 273-279.

9. Jiang H, Du MQ Huang W, Peng B, Bian Z, et al. (2011) The prevalence of and risk factors for non-carious cervical lesions in adults in Hubei Province, China. Community Dent Health 28(1): 22-28.

10. Que K, Guo B, Jia Z, Chen Z, Yang J, et al. (2013) A crosssectional study: non-carious cervical lesions, cervical dentine hypersensitivity and related risk factors. J Oral Rehabil 40(1): 24-32.

11. Wood I, Jawad Z, Paisley C, Brunton P (2008) Noncarious cervical tooth surface loss: A literature review. J Dent 36(10): 759-766.

12. Lyttle HA, Sidhu N, Smyth B (1998) A study of the classification and treatment of noncarious cervical lesions by general practitioners. J Prosthet Dent 79(3): 342-346.

13. Khan F, Young WG, Shahabi S, Daley TJ (1999) Dental cervical lesions associated with occlusal erosion and attrition. Aust Dent J 44(3): 176-186.

14. Ahmed H, Durr E Sadaf, Rahman M (2009) Factors associated with Non-Carious Cervical Lesions (NCCLs) in teeth. J Coll Physicians Surg Pak 19(5): 279-282.

15. Hand JS, Hunt RJ, Reinhardt JW (1986) The prevalence and treatment implications of cervical abrasion in the elderly. Gerodontics 2(5): 167-170.

16. Levitch L, Bader J, Shugars D, Heymann H (1994) Noncarious cervical lesions. Journal of Dentistry 22(4): 195207.

17. Nascimento MM, Gordan VV, Qvist V, Bader JD, Rindal
BD, et al. (2011) Dental Practice-Based Research Network Collaborative Group Restoration of noncarious tooth defects by dentists in The Dental Practice-Based Research Network. J Am Dent Assoc 142(12): 13681375.

18. Bader J, Levitch L, Shugars D, Heymann H, McClure F (1993) How Dentists Classified and Treated Non-Carious Cervical Lesions. J Am Dent Assoc 124(5): 46-54.

19. Pecie R, Krejci I, Godoy FG (2011) Non-carious cervical lesions (NCCL)-A clinical concept based on the literature review. Part 2: Restoration. Am J Dent 24(3): 183-191.

20. Bader J, McClure F, Scurria M, Shugars D, Heymann H (1996) Case-control study of non-carious cervical lesions. Community Dent Oral Epidemiol 24(4): 286291.

21. Grippo J, Simring M, Coleman T (2011) Abfraction, Abrasion, Biocorrosion, and the Enigma of Noncarious Cervical Lesions: A 20-Year Perspective. J Esthet Restor Dent 24(1): 10-23.

22. (2017) NHS Dental Statistics for England 2016-2017, NHS Digital.

23. Kelley K, Clark B, Brown V, Sitzia J (2003) Good practice in the conduct and reporting of survey research. Int J Qual Health Care 15(3): 261-266.

24. Loomba K, Bains R, Bains VK, Loomba A (2014) Proposal for clinical classification of multifactorial noncarious cervical lesions. Gen Dent 62(3): 39-44.

25. Nascimento M, Dilbone D, Pereira P, Geraldeli S, Delgado A, et al. (2016) Abfraction lesions: etiology, diagnosis, and treatment options. Clin, Cosmet Investig 8: 79-87. 